\title{
Total and Cototal Domination Number of Some Zero Divisor Graph
}

\author{
S. Shipiya Raj Shree, V. Maheswari, R. Rajeswari
}

\begin{abstract}
The concept of zero divisor graphs of a commutative ring leads to various research topics since it relates both Ring theory and Graph theory. Domination in graphs has its own development in each field it enters. In this paper, We have given few results on Total and cototal domination number of some zero divisor graph on direct product of commutative rings.
\end{abstract}

Keywrod: zero divisor graphs, Ring theory and Graph theory.

\section{INTRODUCTION}

Let $\mathrm{R}$ be a commutative ring (with identity 1 ) and let $\mathrm{Z}(\mathrm{R})$ be its set of zero-divisors. We associate a (simple) $\Gamma(\mathrm{R})$ to $\mathrm{R}$ with vertices $\mathrm{Z}(\mathrm{R})^{*}=\mathrm{Z}(\mathrm{R})-\{0\}$, the set of non-zero zero-divisor of $R$, and for distinct $x, y \in Z(R)^{*}$, the vertices $x$ and $y$ are adjacent iff $x y=0$. I. Beck introduced Zero divisor graph of a commutative ring. The graphic properties of $\Gamma(\mathrm{R})$ and the theoretic properties of $\mathrm{R}$ can be learn together from its zero divisor graph. Dominating sets form an important research area of Graph theory. B. D. Acharya, E. Sampathkumar and H. B. Waliker are some Indian Mathematians whose contribution in the study of domination in graphs is remarkable. Total domination number was introduced by E.J.Cockayna, R.M.Dawes and S.T.Hedetniemi in the year 1980. In the year 1999, the concept of cototal domination number was defined by V.R.Kulli, B.Janajiram, Radha R.Iyer. The total domination number and cototal domination of a zero divisor graph was found in [7] and [1]. In this paper, we have extended their work to find Total and cototal domination number of some zero divisor graph of direct product on Commutative rings.

Definition 1.1 A nonempty set $\mathrm{R}$ together with two binary operations denoted by " + " and "*" are called addition and multiplication which satisfy

the following axioms is called a ring.

i) $(\mathrm{R},+)$ ia an abelian group.

ii) ":" is an associative binary operation on $\mathrm{R}$.

iii) $\mathrm{a} *(\mathrm{~b}+\mathrm{c})=\mathrm{a} * \mathrm{~b}+\mathrm{a} * \mathrm{c}$ and $(\mathrm{a}+\mathrm{b}) * \mathrm{c}=\mathrm{a} * \mathrm{c}+\mathrm{b} * \mathrm{c}$ for all a,b,c $\in$ R.

A ring $\mathrm{R}$ is said to be commutative if $\mathrm{ab}=\mathrm{ba}$ for $\mathrm{all} \mathrm{a} ; \mathrm{b} \in \mathrm{R}$.

Revised Manuscript Received on October 22, 2019

S. Shipiya Raj Shree, M. Phil Scholar, PG and Research Department of Mathematics, A.P.C Mahalaxmi College for Women, Thoothukudi

V. Maheswari, PG and Research Department of Mathematics, A.P.C Mahalaxmi College for Women, Thoothukudi

R. Rajeswari, PG and Research Department of Mathematics, A.P.C Mahalaxmi College for Women, Thoothukudi

E.mail: shipiyarajshree@gmail.com, maheswari@apcmcollege.ac.in, rajeswari@apcmcollege.ac.in
Definition 1.2 Let $\mathrm{R}$ be a ring. A non-zero element $\mathrm{a} \in \mathrm{R}$ is said to be a zero-divisor if there exists a non-zero element $b$ $\epsilon \mathrm{R}$ such that $\mathrm{ab}=0$ or $\mathrm{ba}=0$.

Definition 1.3 Let $\mathrm{G}(\mathrm{V}, \mathrm{X})$ be simple connected graph. A subet $\mathrm{S} \subseteq \mathrm{V}$ is called a dominating set if every vertex in $\mathrm{V}$ $\mathrm{S}$ is adjacent to a vertex in $\mathrm{S}$. The minimum cardiality of a dominating set in $\mathrm{G}$ is called the dominating number of $\mathrm{G}$.

Definition 1.4 Let $G$ be a graph without isolated vertices. A dominating set $\mathrm{D}$ of $\mathrm{G}$ is a total dominating set if the induced subgraph of $\langle\mathrm{D}\rangle$ contains no isolated vertices. (i.e)A total dominating set $\mathrm{D}$ is the subset of $\mathrm{V}$ such that every vertex $\mathrm{v}$ is adjacent to some vertex in $\mathrm{D}$. The minimum cardinality of total dominating set of $\mathrm{G}$ is called the total domination number of $\mathrm{G}$ and it is denoted by $\gamma_{t}(\mathrm{G})$.

Definition 1.5 A dominating set $D$ of $G$ is a cototal dominating set if every vertex $v \in \mathrm{V}-\mathrm{D}$ is not an isolated vertex in $\langle\mathrm{V}-\mathrm{D}\rangle$. The cototal dominating number $\gamma_{c t}(\mathrm{G})$ of $\mathrm{G}$ is the minimum cardinality of a cototal dominting set.

\section{TOTAL DOMINATION NUMBER OF SOME ZERO- DIVISOR GRAPH}

Here we have found total domination number of some zero divisor graph.

Theorem $2.1 \gamma_{t}\left(\Gamma\left(\mathbb{Z}_{2} \times \mathbb{Z}_{p}\right)\right)=2$

Proof: Let the vertex set of $\Gamma\left(\mathbb{Z}_{2} \times \mathbb{Z}_{p}\right)$ be V $\left(\Gamma\left(\mathbb{Z}_{2} \times \mathbb{Z}_{p}\right)\right)=\{(0,1), \ldots,(0, \mathrm{p}-1),(1,0)\}$ as $\left\{v_{1}, v_{2}, \ldots, v_{p}\right\}$. Clearly $v_{p}$ is adjacent to all other vertices. Therefore, a dominating set is $\left\{v_{p}\right\}$. Let $\mathrm{V}_{1}=$ $\left\{v_{1}, v_{2}, \ldots, v_{p-1}\right\}$ Thus any one vertex from $\mathrm{V}_{1}$ together with $v_{p}$ becomes total dominating set of $\Gamma\left(\mathbb{Z}_{2} \times \mathbb{Z}_{p}\right)$. Hence $\gamma_{t}\left(\Gamma\left(\mathbb{Z}_{2} \times \mathbb{Z}_{p}\right)\right)=2$.

Theorem 2.2 $\gamma_{t}\left(\Gamma\left(\mathbb{Z}_{2} \times \mathbb{Z}_{2 p}\right)\right)=2$, where $\mathrm{p} \geq 5$

Proof: Let $\mathrm{G}=\Gamma\left(\mathbb{Z}_{2} \times \mathbb{Z}_{2 p}\right)$ and

$\mathrm{V}\left(\Gamma\left(\mathbb{Z}_{2} \times \mathbb{Z}_{2 p}\right)\right)=\{(0,1), \ldots$,

$(0,2 p-1),(1,0)\}$ as $\left\{v_{1}, v_{2}, \ldots, v_{2 p}\right\}$. Clearly $v_{2 p}$ is adjacent to all other vertices. Therefore, a dominating set

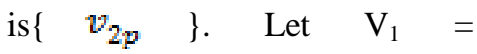


$\{(0,1),(0,2) \ldots,(0,2 p-1)\}$. Thus any one vertex from $V_{1}$ together with $v_{2 p}$ becomes total dominating set of $\mathrm{G}$. Hence $\gamma_{t}\left(\Gamma\left(\mathbb{Z}_{2} \times \mathbb{Z}_{2 p}\right)\right)=2$

Theorem $2.3 \gamma_{t}\left(\Gamma\left(\mathbb{Z}_{2} \times \mathbb{Z}_{3 p}\right)\right)=2$, where $\mathrm{p} \geq 5$

Proof: Let $\mathrm{G}=\Gamma\left(\mathbb{Z}_{2} \times \mathbb{Z}_{3 p}\right)$ and

$\mathrm{V}=\{(0,1), \ldots,(0,3 \mathrm{p}-1),(1,0)\}$ as $\left\{v_{1}, v_{2}, \ldots, v_{3 p}\right\}$. Clearly

$v_{3 p}$ is adjacent to all other vertices. Therefore, a dominating set is $\left\{v_{3 p}\right\}$. Let $\mathrm{V}_{1}=\{(0,1),(0,2) \ldots,(0,3 \mathrm{p})\}$. Thus any one vertex from $\mathrm{V}_{1}$ together with $v_{3 p}$ becomes total dominating set of $\mathrm{G}$. Therefore $\gamma_{t}\left(\Gamma\left(\mathbb{Z}_{2} \times \mathbb{Z}_{3 p}\right)\right)=2$.

Theorem $2.4 \gamma_{t}\left(\Gamma\left(\mathbb{Z}_{2} \times \mathbb{Z}_{4 p}\right)\right)=2$, where $\mathrm{p} \geq 5$

Proof: Let

$\mathrm{V}=\{(0,1), \ldots,(0,4 \mathrm{p}-1),(1,0)\}$ as $\left\{v_{1}, v_{2}, \ldots, v_{4 p}\right\}$. Clearly $v_{4 p}$ is adjacent to all other vertices. Therefore, a dominating set is $\left\{v_{4 p}\right\}$. Let $\mathrm{V}_{1}=\{(0,1),(0,2) \ldots,(0,4 \mathrm{p}-1)\}$. Thus any one vertex from $\mathrm{V}_{1}$ together with $\left\{v_{4 p}\right\}$ becomes total dominating set of $\Gamma\left(\mathbb{Z}_{2} \times \mathbb{Z}_{4 p}\right)$.

Therefore $\gamma_{t}\left(\Gamma\left(\mathbb{Z}_{2} \times \mathbb{Z}_{4 p}\right)\right)=2$.

Theorem $2.5 \gamma_{t}\left(\Gamma\left(\mathbb{Z}_{p} \times \mathbb{Z}_{q}\right)\right)=2$

Proof: Let $\mathrm{V}\left(\Gamma\left(\mathbb{Z}_{p} \times \mathbb{Z}_{q}\right)\right)=\{(0,1), \ldots,(0, \mathrm{q}-1),(1$, $0), \ldots,(p-1,0)\}$. Clearly $\mathbb{Z}_{p} \times \mathbb{Z}_{q}$ is a complete bipartite graph. Now let us split the vertex set into two set $\mathrm{V}_{1}=\{(1$, $0), \ldots,(\mathrm{p}-1,0)\}$ and $\mathrm{V}_{2}=\{(0,1), . .,(0, \mathrm{q}-1)\}$. Let us choose any one point from $\mathrm{V}_{1}$ and any one point from a set $\mathrm{V}_{2}$, this is a dominating set. It is also total dominating set. Hence $\gamma_{t}\left(\Gamma\left(\mathbb{Z}_{p} \times \mathbb{Z}_{q}\right)\right)=2$.

Theorem 2.6 $\gamma_{t}\left(\Gamma\left(\mathbb{Z}_{2} \times \mathbb{Z}_{p^{2}}\right)\right)=2$

Proof: Let

$\mathrm{V}\left(\Gamma\left(\mathbb{Z}_{2} \times \mathbb{Z}_{p^{2}}\right)\right)=\left\{(0,1), \ldots,\left(0, \mathrm{p}^{2}-1\right),(1,0)\right\}=$ $\left\{v_{1}, v_{2}, \ldots, v_{p}{ }^{2}\right\}$

Clearly $v_{p^{2}}$ is adjacent to all the other vertices. Therefore, a dominating set $\mathrm{D}$ is $\left\{v_{p}{ }^{2}\right\}$. Let $\mathrm{V}_{1}=\left\{v_{1}, v_{2}, \ldots, v_{p^{2}-1}\right\}$.

Thus any one vertex from $\mathrm{V}_{1}$ together with $v_{p}$ becomes total dominating set of

$\gamma_{t}\left(\Gamma\left(\mathbb{Z}_{2} \times \mathbb{Z}_{p^{2}}\right)\right)$. Hence $\gamma_{t}\left(\Gamma\left(\mathbb{Z}_{2} \times \mathbb{Z}_{p^{2}}\right)\right)=2$

Theorem $2.7 \gamma_{t}\left(\Gamma\left(\mathbb{Z}_{p q^{2}}\right)\right)=2 ; \mathrm{p} ; \mathrm{q}>2$ and $\mathrm{p}<\mathrm{q}^{2}$, p, q are distinct primes.

Proof: Let us partition the vertex set into 4 sets as multiples of $\mathrm{p}, \mathrm{q}, \mathrm{pq}$ and $\mathrm{q}^{2}$ respectively.

$\mathrm{V}_{1}=\left\{\mathrm{p}, 2 \mathrm{p}, \ldots,(\mathrm{q}-1) \mathrm{p},(\mathrm{q}+1) \mathrm{p}, \ldots, \quad\left(\mathrm{q}^{2}-1\right) \mathrm{p}\right\}$
$\mathrm{V}_{2}=\{\mathrm{q}, 2 \mathrm{q}, \ldots,(\mathrm{p}-1) \mathrm{q},(\mathrm{p}+1) \mathrm{q}, \ldots, \quad((\mathrm{q}-1) \mathrm{p}-1) \mathrm{q},((\mathrm{q}-1) \mathrm{p}$

$+1) \mathrm{q}, \ldots,(\mathrm{pq}-1) \mathrm{q}\}$

$\mathrm{V}_{3}=\{\mathrm{pq}, 2 \mathrm{pq}, \ldots,(\mathrm{q}-1) \mathrm{pq}\}$

$\mathrm{V}_{4}=\left\{\mathrm{q}^{2} ; 2 \mathrm{q}^{2}, \ldots,(\mathrm{p}-1) \mathrm{q}^{2}\right\}$

The vertices in $V_{3}$ are adjacent to all vertices in $V_{2}$ and $V_{4}$. Also $\mathrm{V}_{1}$ and $\mathrm{V}_{4}$ forms a complete bipartite graph. We can choose a vertex from $\mathrm{V}_{4}$ and a vertex from $\mathrm{V}_{4}$. These two vertices form total dominating set. Hence

$\gamma_{t}\left(\Gamma\left(\mathbb{Z}_{p q^{2}}\right)\right)=2$

Theorem $2.8 \gamma_{t}\left(\Gamma\left(\mathbb{Z}_{p} s\right)\right)=1$

Proof: Let

$\mathrm{V}=\left\{\mathrm{p}, 2 \mathrm{p}, \ldots,(\mathrm{p}-1) \mathrm{p}, \mathrm{p}^{2},(\mathrm{p}+1) \mathrm{p}, \ldots, \quad\left(\mathrm{p}^{2}-1\right) \mathrm{p}\right\}$

$\mathrm{V}_{1}=\left\{\mathrm{p}^{2} ; 2 \mathrm{p}^{2}, \ldots,(\mathrm{p}-1) \mathrm{p}^{2}\right\}$

The vertex set $\mathrm{V}_{1}$ form a complete graph $\mathrm{K}_{\mathrm{p}-1}$ and also all the vertices in this set are adjacent to all other vertices of $\Gamma\left(\mathbb{Z}_{p} s\right)$. Therefore by choosing any one of the vertex in $V_{1}$ together with any other vertices, we will get a total dominating set. Hence $\gamma_{t}\left(\Gamma\left(\mathbb{Z}_{p} \mathrm{~s}\right)\right)=1$.

\section{COTOTAL DOMINATION NUMBER}

In this section, we investigate Cototal domination number for some Zero divisor graphs.

Theorem $3.1 \gamma_{c t}\left(\Gamma\left(\mathbb{Z}_{2} \times \mathbb{Z}_{p}\right)\right)=\mathrm{p}$

Proof: Let the vertex set of $\Gamma\left(\mathbb{Z}_{2} \times \mathbb{Z}_{p}\right)$ be V $\left(\Gamma\left(\mathbb{Z}_{2} \times \mathbb{Z}_{p}\right)\right)=\{(1,0),(0,1), \ldots,(0, \mathrm{p}-1)\}$ as $\left\{v_{1}, v_{2}, \ldots, v_{p}\right\}$.Clearly $\mathrm{v}_{1}$ is adjacent to all other vertices. Therefore, a dominating set contains $\left\{\mathrm{v}_{1}\right\}$. Then all other vertices becomes an isolated vertices in $\mathrm{V}-\mathrm{D}$.

Now, let $\mathrm{D}=\left\{v_{1}, v_{2}, \ldots, v_{p}\right\}$. Thus $\mathrm{D}$ becomes cototal dominating set of

$\Gamma\left(\mathbb{Z}_{2} \times \mathbb{Z}_{p}\right)$. Hence $\gamma_{c t}\left(\Gamma\left(\mathbb{Z}_{2} \times \mathbb{Z}_{p}\right)\right)=\mathrm{p}$

Theorem $3.2 \gamma_{c t}\left(\Gamma\left(\mathbb{Z}_{2} \times \mathbb{Z}_{2 p}\right)\right)=p$, where $\mathrm{p} \geq 5$

Proof: Let $\mathrm{G}=\Gamma\left(\mathbb{Z}_{2} \times \mathbb{Z}_{2 p}\right)$ and

$\mathrm{V}\left(\Gamma\left(\mathbb{Z}_{2} \times \mathbb{Z}_{2 p}\right)\right)=\{(0,1), \ldots$,

$(0,2 \mathrm{p}-1),(1,0)\}$ as $\left\{v_{1}, v_{2}, \ldots, v_{2 p}\right\}$.

The vertex $v_{2 p}$ is adjacent to all other vertices. Now let us split the vertex set into two set $\mathrm{A}=\left\{\mathrm{v}_{2 \mathrm{i}-1} / 1 \leq \mathrm{i} \leq \mathrm{p}-1\right\}$ and $\mathrm{B}=\left\{\mathrm{v}_{2 \mathrm{i}} / 1 \leq \mathrm{i} \leq \mathrm{p}-1\right\}$

The vertices in $\mathrm{B}$ are adjacent to $\mathrm{v}_{\mathrm{p}} \in \mathrm{A}$. In $\langle\mathrm{V}-\mathrm{D}\rangle$, we will get all vertices of $A$ except $v_{p}$ as isolated vertices. So, we include all these isolated vertices in the cototal dominating set. Therefore $|D|=1+p-1=p$, where $D$ is the cototal dominating set. Hence $\gamma_{c t}\left(\Gamma\left(\mathbb{Z}_{2} \times \mathbb{Z}_{2 p}\right)\right)=p$.

Published By: Blue Eyes Intelligence Engineering 
Theorem $3.3 \gamma_{c t}\left(\Gamma\left(\mathbb{Z}_{2} \times \mathbb{Z}_{3 p}\right)\right)=2 p-1$, where $\mathrm{p} \geq 5$ Proof: Let

Let $\mathrm{G}=\Gamma\left(\mathbb{Z}_{2} \times \mathbb{Z}_{3 p}\right)$ and

$\mathrm{V}=\{(0,1), \ldots,(0,3 \mathrm{p}-1),(1,0)\}$ as $\left\{v_{1}, v_{2}, \ldots, v_{3 p}\right\}$. Clearly

$v_{3 p}$ is adjacent to all other vertices. Therefore, a dominating set is $\left\{v_{3 p}\right\}$. Now let us consider a vertex set $\mathrm{V}_{1}=\left\{\mathrm{v}_{3 \mathrm{i}} / 1 \leq \mathrm{i}\right.$ $\leq \mathrm{p}-1\}$. The vertices $\mathrm{v}_{\mathrm{p}}, \mathrm{v}_{2 \mathrm{p}}$ are adjacent to all vertices of $\mathrm{V}_{1}$. Clearly the vertices in $\mathrm{V}_{1}$ and $\left\{\mathrm{v}_{\mathrm{p}}, \mathrm{v}_{2 \mathrm{p}}\right\}$ are not isolated in $\langle\mathrm{V}$-D $\rangle$. So, we include all isolated vertices in the cototaldominating set. Therefore $\gamma_{c t}\left(\Gamma\left(\mathbb{Z}_{2} \times \mathbb{Z}_{3 p}\right)\right)=3 p$ $-[(\mathrm{p}-1)+2]=2 \mathrm{p}-1$.

Theorem $3.4 \gamma_{c t}\left(\Gamma\left(\mathbb{Z}_{2} \times \mathbb{Z}_{4 p}\right)\right)=2 p-1$, where $p \geq 5$ Proof: Let

$\mathrm{V}=\{(0,1), \ldots,(0,4 \mathrm{p}-1),(1,0)\}$ as $\left\{v_{1}, v_{2}, \ldots, v_{4 p}\right\}$. Clearly $v_{4 p}$ is adjacent to all other vertices. Therefore, a dominating set is $\left\{v_{4 p}\right\}$. Now let us consider a vertex set $\mathrm{V}_{1}=\left\{\mathrm{v}_{2 \mathrm{i}} / 1 \leq \mathrm{i}\right.$ $\leq \mathrm{p}-1, \mathrm{p}+1 \leq \mathrm{i} \leq 2 \mathrm{p}-1\}$. The vertex $\mathrm{v}_{2 \mathrm{p}}$ is adjacent to all vertices of $V_{1}$. Clearly the vertices in $V_{1}$ and $v_{2 p}$ are not isolated in $\langle\mathrm{V}-\mathrm{D}\rangle$. Also $\mathrm{v}_{\mathrm{p}}, \mathrm{v}_{3 \mathrm{p}}$ are adjacent to all vertices in the set $\left\{\mathrm{v}_{4 \mathrm{i}} / 1 \leq \mathrm{i} \leq \mathrm{p}-1\right\}$. So, we include all other isolated vertices in the cototal dominating set. Therefore $\gamma_{c t}\left(\Gamma\left(\mathbb{Z}_{2} \times \mathbb{Z}_{4 p}\right)\right)=4 \mathrm{p}-[2 \mathrm{p}-1+2]=2 \mathrm{p}-1$

Theorem $3.5 \gamma_{c t}\left(\Gamma\left(\mathbb{Z}_{p} \times \mathbb{Z}_{q}\right)\right)=2$

Proof: Let V $\left(\Gamma\left(\mathbb{Z}_{p} \times \mathbb{Z}_{q}\right)\right)=\{(0,1), \ldots,(0, \mathrm{q}-1),(1,0)$, $\ldots,(\mathrm{p}-1,0)\}$. Clearly $\Gamma\left(\mathbb{Z}_{p} \times \mathbb{Z}_{q}\right)$ is a complete bipartite graph. Now let us split the vertex set into two set $\mathrm{V}_{1}=\{(1$, $0), \ldots,(p-1,0)\}$ and $V_{2}=\{(0,1), \ldots,(0 q-1)\}$. Let us choose any one point from $\mathrm{V}_{1}$ and any one point from a set $\mathrm{V}_{2}$, this is adominating set. It is also cototal dominating set. Hence $\gamma_{c t}\left(\Gamma\left(\mathbb{Z}_{p} \times \mathbb{Z}_{q}\right)\right)=2$.

Theorem 3.6 $\gamma_{c t}\left(\Gamma\left(\mathbb{Z}_{2} \times \mathbb{Z}_{p^{2}}\right)\right)=p^{2}-\mathrm{p}+1$

Proof: Let

$\mathrm{V}\left(\Gamma\left(\mathbb{Z}_{2} \times \mathbb{Z}_{p^{2}}\right)\right)=\left\{(0,1), \ldots,\left(0, p^{2}-1\right),(1,0)\right\}=$ $\left\{v_{1}, v_{2}, \ldots, v_{p}{ }^{2}\right\}$. Clearly $v_{p}$ is adjacent to all other vertices. Therefore, a dominating set $\mathrm{D}$ contains $v_{p^{2}}$. There is a subgraph $\mathrm{K}_{\mathrm{p}-1}$ in $\Gamma\left(\mathbb{Z}_{2} \times \mathbb{Z}_{p^{2}}\right)$. This $\mathrm{p}-1$ vertices are connected in $\langle\mathrm{V}-\mathrm{D}\rangle$. Let us include all other isolated vertices in the

cototal dominating set. Hence $\gamma_{c t}\left(\Gamma\left(\mathbb{Z}_{2} \times \mathbb{Z}_{p^{2}}\right)\right)=p^{2}$ $\mathrm{p}+1$
Theorem $3.7 \gamma_{c t}\left(\Gamma\left(\mathbb{Z}_{p q^{2}}\right)\right)=2$; p; $>2$ and $\mathrm{p}<q^{2}$; $\mathrm{q}$ are distinct primes.

Proof: Let us partition the vertex set into 4 sets as multiples of $\mathrm{p}$; $\mathrm{q} ; \mathrm{pq}$; and $\mathrm{q}^{2}$ respectively.

$\mathrm{V}_{1}=\left\{\mathrm{p}, 2 \mathrm{p}, \ldots,(\mathrm{q}-1) \mathrm{p},(\mathrm{q}+1) \mathrm{p}, \ldots, \quad\left(\mathrm{q}^{2}-1\right) \mathrm{p}\right\}$

$\mathrm{V}_{2}=\{\mathrm{q}, 2 \mathrm{q}, \ldots,(\mathrm{p}-1) \mathrm{q},(\mathrm{p}+1) \mathrm{q}, \ldots, \quad((\mathrm{q}-1) \mathrm{p}-1) \mathrm{q},((\mathrm{q}-1) \mathrm{p}$

$+1) \mathrm{q}, \ldots,(\mathrm{pq}-1) \mathrm{q}\}$

$\mathrm{V}_{3}=\{\mathrm{pq}, 2 \mathrm{pq}, \ldots,(\mathrm{q}-1) \mathrm{pq}\}$

$\mathrm{V}_{4}=\left\{\mathrm{q}^{2} ; 2 \mathrm{q}^{2}, \ldots,(\mathrm{p}-1) \mathrm{q}^{2}\right\}$

The vertices in $V_{3}$ are adjacent to all vertices in $V_{2}$ and $V_{4}$. Also $\mathrm{V}_{1}$ and $\mathrm{V}_{4}$ forms a complete bipartite graph. We can choose a vertex from $V_{4}$ and a vertex from $V_{3}$. These two vertices form cototal dominating set. Hence

$\gamma_{c t}\left(\Gamma\left(\mathbb{Z}_{p q^{2}}\right)\right)=2$.

Theorem $3.8 \gamma_{c t}\left(\Gamma\left(\mathbb{Z}_{p} s\right)\right)=1$

Proof: Let

$\mathrm{V}=\left\{\mathrm{p}, 2 \mathrm{p}, \ldots,(\mathrm{p}-1) \mathrm{p}, \mathrm{p}^{2},(\mathrm{p}+1) \mathrm{p}, \ldots, \quad\left(\mathrm{p}^{2}-1\right) \mathrm{p}\right\}$

$\mathrm{V}_{1}=\left\{\mathrm{p}^{2} ; 2 \mathrm{p}^{2}, \ldots,(\mathrm{p}-1) \mathrm{p}^{2}\right\}$

The vertex set $\mathrm{V}_{1}$ form a complete graph $\mathrm{K}_{\mathrm{p}-1}$ and also all the vertices in this set are adjacent to all other vertices of $\Gamma\left(\mathbb{Z}_{p}\right.$ s $)$. Therefore by choosing any one of the vertex in $\mathrm{V}_{1}$ we will get a cototal dominating set. Hence

$\gamma_{c t}\left(\Gamma\left(\mathbb{Z}_{p} s\right)\right)=1$

\section{REFERENCES}

[1]. Ananthi K., Selvi N., Cototal Domination Number of a Zero Divisor Graph, IJMAA ISSN:2347-1557, Vol-V, Issue 2-C(2017), 339-343.

[2]. Anderson, D.D and Naseer, M., Beck's coloring of a commutative ring, J. Algebra 159 (1993), 500-514.

[3]. Anderson, David F., and Livingston, Philip S., The Zero-divisor graph of commutative ring, J. Algebra 217 (1999), 434-447.

[4]. Axtell M., Stickles J., andWarfel J., Zero-divisor graphs of direct products of commutative rings, Houston journal of Mathematics, January 2006.

[5]. Beck I., Coloring of Commutative rings, J. Algebra 116 (1988), 208-226.

[6]. Bollabas B., Graph Teory, An Introductory Course, Springer- Verlag, New York. 1979.

[7]. Budadoddi K., Ramakrishna R., and Mallikarjuna Reddy A., Some Results on Total Domination Number of Zero Divisor Graph, IJIRSET(July-2017), ISSN(online): 2319-8753.

[8]. Ganesan N., Properties of rings with a finite number of zero-divisors, Math. Ann. 157 (1964), 215-218.

[9]. Ganesan N., Properties of rings with a finite number of zero-divisors, II, Math. Ann. 161 (1965), 241-24. 